DE

M E D I C I N A

T R O P I C A L

$\mathrm{DE}$

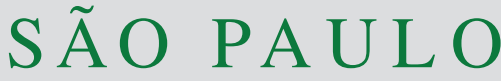

JOURNAL OF THE SÃO PAULO INSTITUTE OF TROPICAL MEDICINE

'Universidade de Brasília, Faculdade de Medicina, Programa de Pós-Graduação em Ciências Médicas, Brasília, Brazil

${ }^{2}$ Hospital Universitário de Brasília, Brasília, Brazil

${ }^{3}$ Universidade de Brasília, Faculdade de Medicina, Grupo de Diagnóstico Dermatológico, Brasília, Brazil

${ }^{4}$ Universidade de Brasília, Faculdade de Medicina, Laboratório de Dermatomicologia, Brasília, Brazil

${ }^{5}$ Case Western Reserve University, Department of Dermatology, Cleveland, Ohio, USA

${ }^{6}$ IRCCS Istituto Ortopedico Galeazzi, Dermatologia Clinica, Milan, Italy

${ }^{7}$ Università degli Studi di Milano, Dipartimento di Scienze Biomediche, Chirurgiche e Odontoiatriche, Milan, Italy

${ }^{8}$ Universidade de Brasília, Faculdade de Ciências da Saúde, Programa de PósGraduação em Ciências da Saúde, Brasília, Brazil

'Universidade de São Paulo, Faculdade de Medicina de Ribeirão Preto, Departamento de Clínica Médica, Programa de PósGraduação em Clínica Médica, Ribeirão Preto, São Paulo, Brazil

${ }^{10}$ Universidade de Brasília, Faculdade de Medicina, Núcleo de Medicina Tropical, Programa de Pós-Graduação em Medicina Tropical, Brasília, Brazil

Correspondence to: Ciro Martins Gomes Hospital Universitário de Brasília, Av. L2 Norte, Brasília, Distrito Federal, CEP 70840-901, Brazil

Tel: +5561 2028-5415, Fax: + 5561 3225-6799

E-mail: ciromgomes@gmail.com

Received: 17 January 2020

Accepted: 17 April 2020

\section{A comprehensive systematic review of leishmaniasis in patients undergoing drug-induced immunosuppression for the treatment of dermatological, rheumatological and gastroenterological diseases}

\author{
Patrícia Shu Kurizky ${ }^{1,2,3,4}$, Fernanda Ferraço Marianelli ${ }^{2}$, Mariana Vicente \\ Cesetti ${ }^{1}$, Giovanni Damiani5,6,7, Raimunda Nonata Ribeiro Sampaio ${ }^{1,3,4,8}$, Lais \\ Mitsue Tanaka Gonçalves ${ }^{2}$, Carlos Augusto Felipe de Sousa', Sofia Sales \\ Martins $^{8}$, Sebastian Vernal ${ }^{9}$, Licia Maria Henrique da Mota ${ }^{1,2}$, Ciro Martins \\ Gomes $^{\circledR 1,2,3,4,10}$
}

\section{ABSTRACT}

Immunosuppression is an important risk factor for leishmaniasis. We assessed the clinical profile, geographic distribution and prevalence of leishmaniasis in patients undergoing immunosuppressive therapy for dermatological, rheumatological or gastroenterological autoimmune diseases. We identified relevant studies in PubMed, EMBASE, Scopus, Web of Science and LILACS on July $3^{\text {rd }}, 2018$. We included articles that reported at least one case of leishmaniasis in patients undergoing immunosuppressive treatment for dermatological, rheumatological or gastroenterological diseases. Our protocol was registered in PROSPERO (CRD42018103050). We assessed the quality of the included studies with the Joanna Briggs Institute Critical Appraisal Tool. After the removal of duplicates, 5,431 articles were collected and screened. We included 138 articles; the prevalence of leishmaniasis in six methodologically similar studies varied from three to 1,282 cases per 100,000 patients using anti-TNF $\alpha$ drugs, but the results were significantly heterogeneous . Leishmaniasis in patients treated with immunosuppressive drugs is a health problem mostly reported in European countries bordering the Mediterranean Sea; sporadic activities, such as travelling, seem not to be associated with a significant risk of leishmaniasis, although effective control measures must always be observed.

KEYWORDS: Immunosuppression. Leishmaniasis. Systematic review. Treatment.

\section{INTRODUCTION}

Leishmaniasis is an anthropozoonosis that is caused by several flagellate protozoan species of the genus Leishmania and transmitted by insects of the genus Lutzomyia $^{1}$. The World Health Organization (WHO) includes leishmaniasis among the six most important infectious diseases worldwide ${ }^{2}$. The disease is considered a public health problem in 64 countries, with $90 \%$ of cases concentrated in India, Bangladesh, Nepal, Sudan and Brazil ${ }^{3}$.

Immunosuppression is an important risk factor for the pathogenesis of severe leishmaniasis and reactivation; furthermore, it can alter the response to treatment, resulting in adverse outcomes ${ }^{1}$. Although human immunodeficiency virus (HIV) infection is the most studied example of immunosuppression, other causes of immunosuppression have been increasingly associated with leishmaniasis, mainly 
due to the increasing use of immunomodulatory drugs ${ }^{4}$. These drugs constitute an important basis for the treatment of autoimmune disorders ${ }^{5}$. Immunobiologicals are a relatively modern class of drugs that have revolutionized the control of immune-mediated diseases and are widely used for the treatment of dermatological, rheumatological and gastroenterological conditions. These drugs specifically block important mediators, such as TNF- $\alpha$ and other cytokines, thereby enhancing the risk of infection and of leishmaniasis reactivation.

Although the increased risk of tuberculosis was the initial focus regarding infections in patients treated with immunosuppressive drugs, there are now a substantial number of reports on leishmaniasis, which is usually diagnosed after several months of treatment ${ }^{4}$. In this context, we sought to develop a clinical pathway formed by questions that could be answered as the main objectives of this review and that could elucidate literature gaps for further studies (Figure 1). Focusing on the main objectives, we proposed the following questions: What is the clinical profile and the geographic distribution of leishmaniasis in patients receiving immunosuppressive therapy for rheumatological, dermatological and gastroenterological diseases? What is the frequency of leishmaniasis in patients receiving these specific immunosuppressive therapies? To identify possible literature gaps, we proposed the following questions: do patients exposed to immunosuppressive biologicals have a higher risk of developing leishmaniasis than those under immunosuppression caused by other factors? Do immunosuppressed patients need specific measures to avoid Leishmania infection or leishmaniasis reactivation?

The main objective of the present systematic review was to assess the clinical profile, geographic distribution and prevalence of leishmaniasis in patients using any immunosuppressive agent for the treatment of rheumatological, dermatological or gastroenterological diseases. We also aimed to assess the existing literature gaps that need to be fulfilled as identified in the developed clinical pathway (Figure 1).

\section{MATERIALS AND METHODS}

\section{Search strategy and selection criteria}

The present protocol was registered in PROSPERO (CRD42018103050) on 14 August 2018. A comprehensive

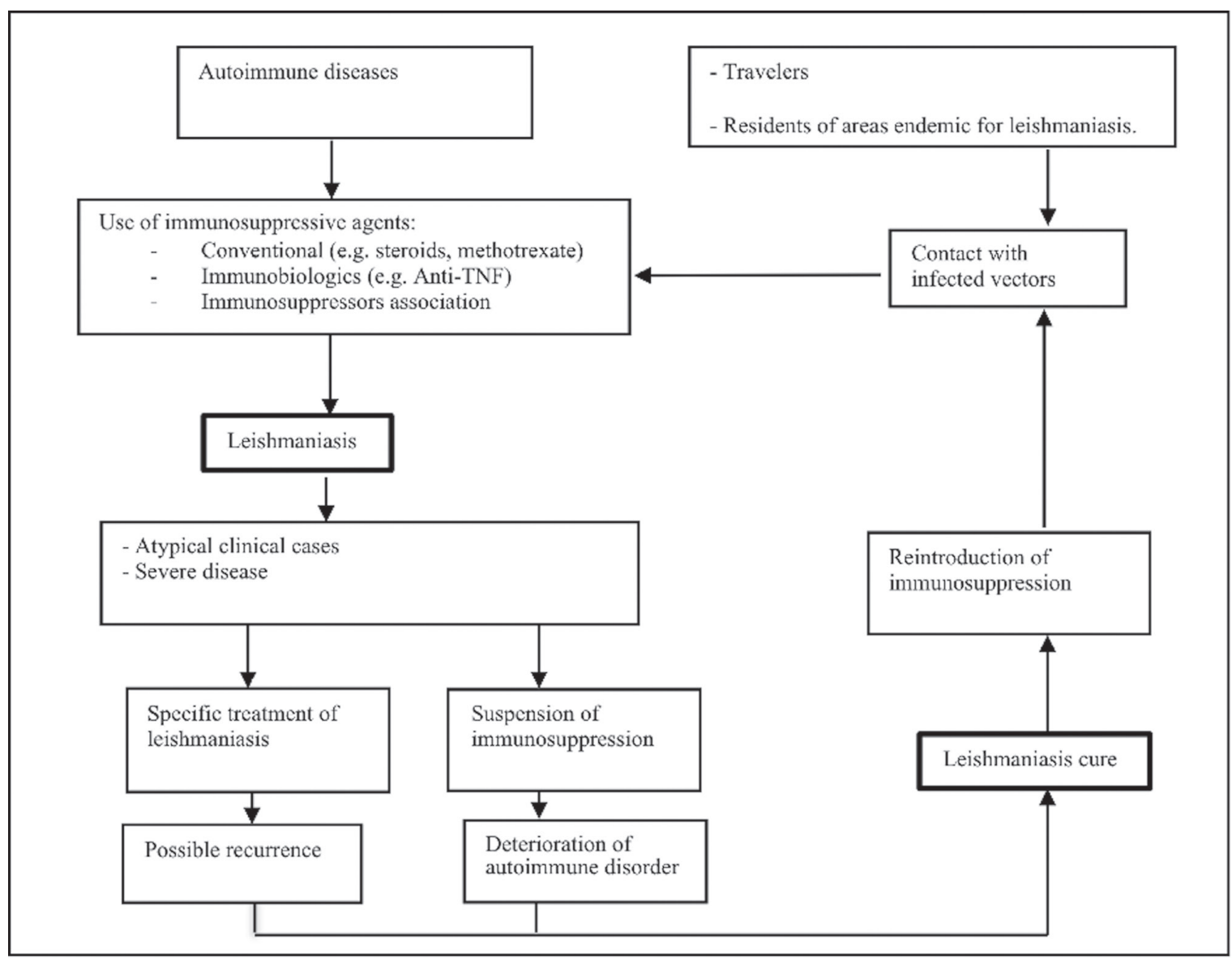

Figure 1 - Current clinical pathway for the occurrence of leishmaniasis in immunosuppressed patients. 
search of the following relevant databases was performed on 3 July 2018: PubMed, EMBASE, Scopus, Web of Science and LILACS (Table 1). Grey literature (conference abstracts and proceedings) were included in the search. No date, language or method restrictions were applied. No additional search for grey literature was performed.

We included any article that reported at least one case of leishmaniasis in patients undergoing treatment with immunosuppressive drugs for dermatological, rheumatological or gastroenterological diseases, which are medical conditions frequently treated with immunobiologicals, a relatively new and widely used class of medication compared to conventional immunosuppressive agents (e.g., cyclosporine and methotrexate).

We excluded articles in which leishmaniasis occurred before the immunosuppressive treatment, as a result of an incorrect diagnosis; articles in which immunosuppression was not a result of treatment for a rheumatological, dermatological or gastroenterological disease and articles that diagnosed leishmaniasis by only serological or molecular examinations in asymptomatic patients.

\section{Data extraction}

After database searching, all the retrieved references were exported to EPPI-Reviewer 4 Version 4.6.4.0 (EPPI Centre, London, England), and duplicates were removed with the aid of an automatic tool included in the program. Two independent reviewers (PK and CG) screened the titles and abstracts and, for the articles selected during the initial screening process, the full texts were examined. Disagreements were resolved by a third reviewer (MC).

Two independent reviewers were responsible for data extraction (PK and FM). Disagreements were resolved by a third reviewer (CG). A data-extraction form was developed based on the authors' clinical experience in the use of immunosuppressive agents and the current practical literature. Prior to data extraction, we performed a pilot data extraction with 10 randomly assigned articles to identify any possible obstacles.

\section{Quality assessment}

Case reports and case series were evaluated by two independent reviewers (CG and FM) using the Joanna Briggs Institute Critical Appraisal Tool ${ }^{6}$. Prevalence studies were independently evaluated (CG and FM) using the Joanna Briggs Institute Critical Appraisal Tool for prevalence studies. Disagreements were resolved by consensus.

Table 1 - Search strategy and databases accessed for the systematic review of the literature.

\begin{tabular}{|c|c|}
\hline Accessed databases & Search strategy \\
\hline PUBMED & $\begin{array}{l}\text { (corticosteroid* OR methotrexate OR leflunomide OR cyclosporine OR infliximab OR adalimumab OR } \\
\text { etanercept OR golimumab OR steroid* OR secukinumab OR ustekinumab OR apremilast OR abatacept } \\
\text { OR azathioprine OR mycophenol* OR rituximab OR sulfasalazine OR tocilizumab OR tofacitinib OR } \\
\text { certolizumab*) OR immunos* AND leish* }^{*}\end{array}$ \\
\hline LILACs & 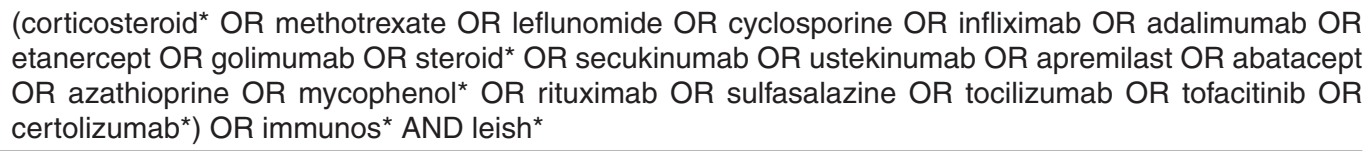 \\
\hline SCOPUS & $\begin{array}{l}\text { (ALL (methotrexate) OR ALL (leflunomide) OR ALL (cyclosporine) OR ALL (infliximab) OR ALL } \\
\text { (adalimumab) OR ALL (etanercept) OR ALL (golimumab) OR steroid* OR secukinumab OR ustekinumab } \\
\text { OR apremilast OR abatacept OR azathioprine OR mycophenol* OR rituximab OR sulfasalazine OR } \\
\text { tocilizumab OR tofacitinib OR certolizumab*) OR immunos* AND leish* }\end{array}$ \\
\hline Web of Science & $\begin{array}{l}\# 1 \mathrm{TS}=\left(\text { corticosteroid }{ }^{\star} \text { OR methotrexate OR leflunomide OR cyclosporine OR infliximab OR adalimumab }\right. \\
\text { OR etanercept OR golimumab OR steroid* OR secukinumab OR ustekinumab OR apremilast OR abatacept } \\
\text { OR azathioprine OR mycophenol* OR rituximab OR sulfasalazine OR tocilizumab OR tofacitinib OR } \\
\text { certolizumab*) } \\
\text { \# } 2 \text { TS= }\left(\text { immunos }^{\star}\right) \\
\text { \# } 3 \text { \#1 OR \#2 } \\
\left.\text { \#4 TI= (leish }{ }^{\star}\right) \\
\text { \#5 \#3 AND \#4 }\end{array}$ \\
\hline EMBASE & $\begin{array}{l}\text { \#1 corticosteroid* OR methotrexate OR leflunomide OR cyclosporine OR infliximab OR adalimumab OR } \\
\text { etanercept OR golimumab OR steroid* OR secukinumab OR ustekinumab OR apremilast OR abatacept } \\
\text { OR azathioprine OR mycophenol* OR rituximab OR sulfasalazine OR tocilizumab OR tofacitinib OR } \\
\text { certolizumab* } \\
\text { \# } 2 \text { leish* ti.ab.kw } \\
\text { \#3 \#1 AND \#2 }\end{array}$ \\
\hline
\end{tabular}




\section{Data analysis}

Firstly, a descriptive analysis of leishmaniasis cases in patients treated with immunosuppressive agents was performed. An epidemiological profile of the affected patients was created by considering the immunosuppressive agents used, the duration of autoimmune disease and the duration of immunosuppression. The geographical distribution of the cases was also compared to the endemicity in each country.

Secondly, all cross-sectional and longitudinal studies were evaluated. We ultimately selected studies that assessed the prevalence of leishmaniasis in patients under immunosuppression. The final model consisted of articles with similar methodologies and risk factor profiles. As leishmaniasis was a secondary outcome in most articles in this group, we could not associate the leishmaniasis cases with the duration of immunosuppression, and we could not calculate the incidence or the risk.

Proportions were calculated, and double arcsine transformations were applied to stabilize the variance. We calculated the overall summary proportion considering within- and between-study variation with the random effects model for the meta-analysis. Calculations were performed and plots were generated with the packages "meta", "metafor" and "weightr" in the program RStudio for Mac (RStudio: Integrated Development for R, RStudio, Inc., Boston, MA, USA) $)^{7-9}$.

\section{RESULTS}

In total, 8,433 articles were initially selected from the referenced databases, and six additional articles were included after reading the references of the included full texts. After the removal of duplicates, the titles and abstracts of 5,431 articles were screened, and the full texts of 293 studies were evaluated. Finally, 138 articles that met the inclusion criteria were selected (Figure 2).

\section{Characteristics of the selected studies}

Most articles $(n=124)$ were case reports or case series. Six population-based surveys of patients with leishmaniasis were also included. Eight articles evaluated the proportion

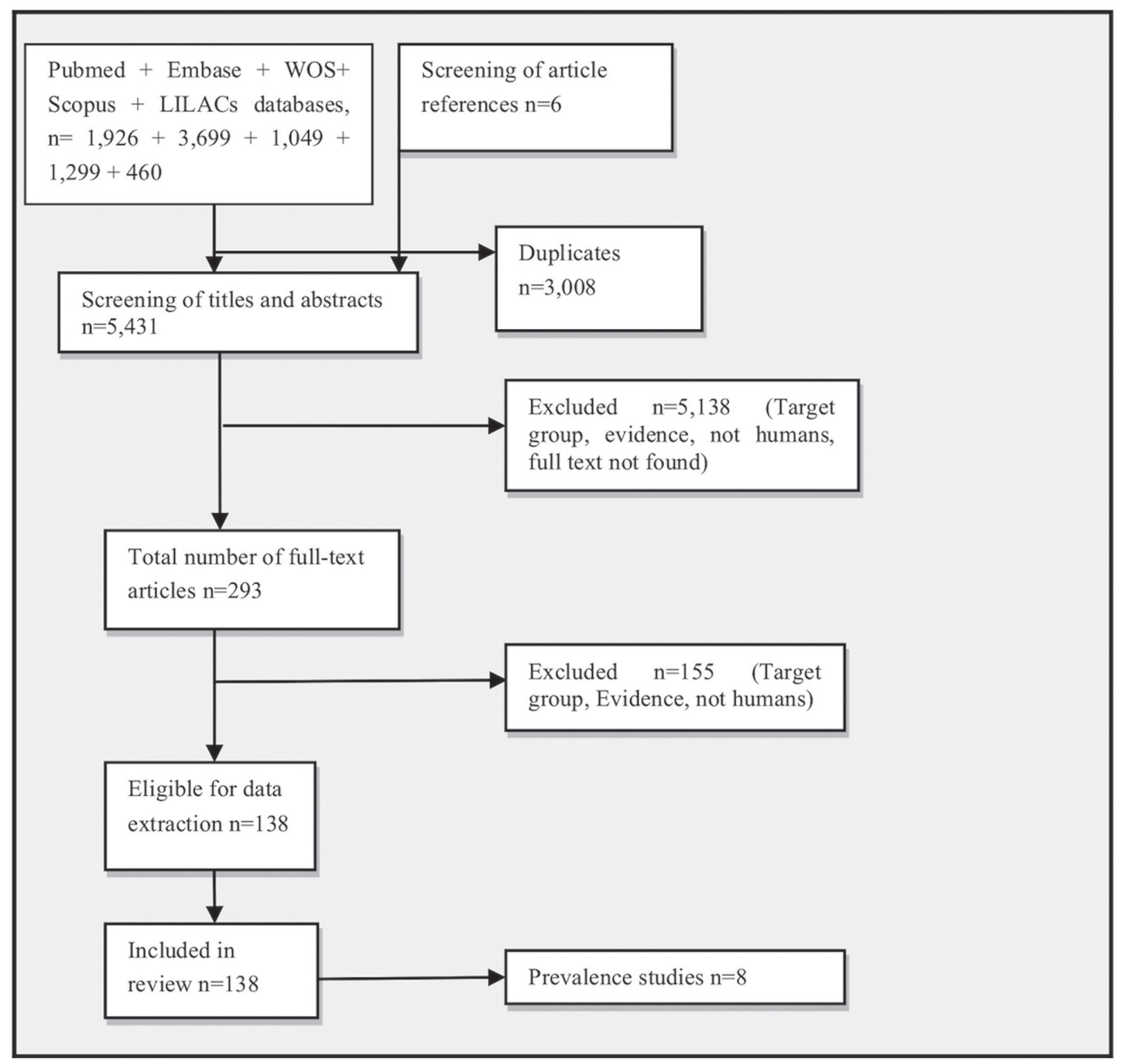

Figure 2 - The flow diagram of the search and selection process of articles. 
of patients with leishmaniasis among patients receiving immunosuppressive agents. Sixteen studies were published as meeting abstracts, while the remainder were journal articles.

None of the selected articles compared the prevalence of leishmaniasis in immunosuppressed patients with a control group of immunocompetent ones, making it impossible to calculate the relative prevalence. In total, 189 immunosuppressed patients developed leishmaniasis.

\section{Leishmaniasis case characteristics}

In immunosuppressed patients, the mean age at the time of leishmaniasis development was 51.63 years, the mean duration of the immunological disease was 12.09 years (range $=1-44$ years, standard deviation $(\mathrm{SD})=9.20)$ and the mean duration of immunosuppression was 5.75 years (range $=0.1$ to 30 years, $\mathrm{SD}=6.14$ ). Forty-one patients used systemic corticosteroids, 96 used immunobiologicals, 42 used methotrexate and 3 used azathioprine (in seven cases, the immunosuppressive drugs were not specified).

Most patients $(\mathrm{n}=105)$ developed visceral leishmaniasis, 65 patients developed cutaneous leishmaniasis and 14 patients developed mucocutaneous leishmaniasis. Only 10 patients reported having had contact with leishmaniasis patients or dogs known to have leishmaniasis. Considering the clinical picture, only 39 atypical leishmaniasis cases were reported. A Leishmania species known to cause visceral disease (Leishmania infantum) resulted in mucocutaneous leishmaniasis in only one case ${ }^{10}$.

Parasitological diagnostic methods were used in 117 patients, while polymerase chain reaction (PCR) was necessary for diagnosis in only 25 patients. Most identified Leishmania species were Old World species, including Leishmania aethiopica $(\mathrm{n}=2)$, Leishmania donovani $(\mathrm{n}=15)$, Leishmania infantum $(\mathrm{n}=33)$, Leishmania major $(\mathrm{n}=3)$, Leishmania tropica $(\mathrm{n}=2)$, Leishmania amazonensis $(\mathrm{n}=1)$ and Leishmania braziliensis $(\mathrm{n}=2)$.

Treatment usually consisted of the administration of liposomal amphotericin B ( $\mathrm{n}=89$ cases), and pentavalent antimonials $(\mathrm{n}=52$ cases). Ninety-two patients were cured with only one course of treatment, while 22 needed more than one course of treatment to cure leishmaniasis. In 72 cases, immunosuppression was halted before leishmaniasis-specific treatment was administered, and in 34 cases, immunosuppression was resumed after leishmaniasis was cured.

Only 10 patients had apparent reactivation of latent leishmaniasis, while the vast majority $(n=112)$ were probably infected while on immunosuppressive therapy. Most patients were undergoing immunosuppressive therapy for rheumatological diseases $(\mathrm{n}=152)$;
19 immunosuppressed patients had dermatological conditionsand 18 had gastroenterological conditions. In most cases, the probable location of infection was Europe $(\mathrm{n}=144)$, in countries bordering the Mediterranean Sea (Figure 3). The remaining cases occurred in the Middle East $(n=4)$, Asia $(n=1)$, South America $(n=10)$ and Africa $(n=15)$. Only 24 patients were travellers and only 6 reported occupational risk factors (Figure 3 ).

\section{Quality assessment}

Regarding the eight articles ${ }^{11-18}$ that reported the prevalence of leishmaniasis in patients treated with immunosuppressants, none described the study subjects or settings in detail. No articles mentioned the validation methods used for the identification of the medical condition, any comparison to a known standard, or a reliable way to determine the domain response rate.

\section{Prevalence evaluation}

The eight articles ${ }^{11-18}$ that evaluated the prevalence of leishmaniasis in patients treated with immunosuppressive agents included 68,474 patients and 10 cases were reported in this population (Table 2). Eight patients developed leishmaniasis while taking TNF blockers ${ }^{11,14,17,18}$, one patient developed leishmaniasis while taking an interleukin-1 receptor antagonist (anakinra) ${ }^{15}$, and one patient developed leishmaniasis while being treated for dermatomyositis/polymyositis, a condition that can be treated with steroids, conventional immunosuppressive agents and/or immunobiologicals ${ }^{16}$. Considering only the studies $(n=3)$ that evaluated more than 1,000 participants, the prevalence of leishmaniasis in the immunosuppressed population ranged from 3 to 32 leishmaniasis cases per 100,000 patients using anti-TNF drugs (Table 2).

Six articles were considered methodologically similar (patients using TNF- $\alpha$ blockers) (Table 2 ) and were selected to be included in the meta-analysis; however, a high degree of heterogeneity was detected $\left(\mathrm{I}^{2}=76 \% ; \mathrm{p}<0.001\right)$. This heterogeneity was also detected by visual evaluation of the effect sizes and confidence intervals reported in each individual study (Figure 4). The prevalence of leishmaniasis in the six methodologically similar studies varied from 3 to 1,282 cases per 100,000 patients using anti-TNF- $\alpha$ drugs. The small number of studies $(n=6)$ limited the publication bias analysis ${ }^{19}$.

\section{DISCUSSION}

Studies involving HIV-positive populations have shown 


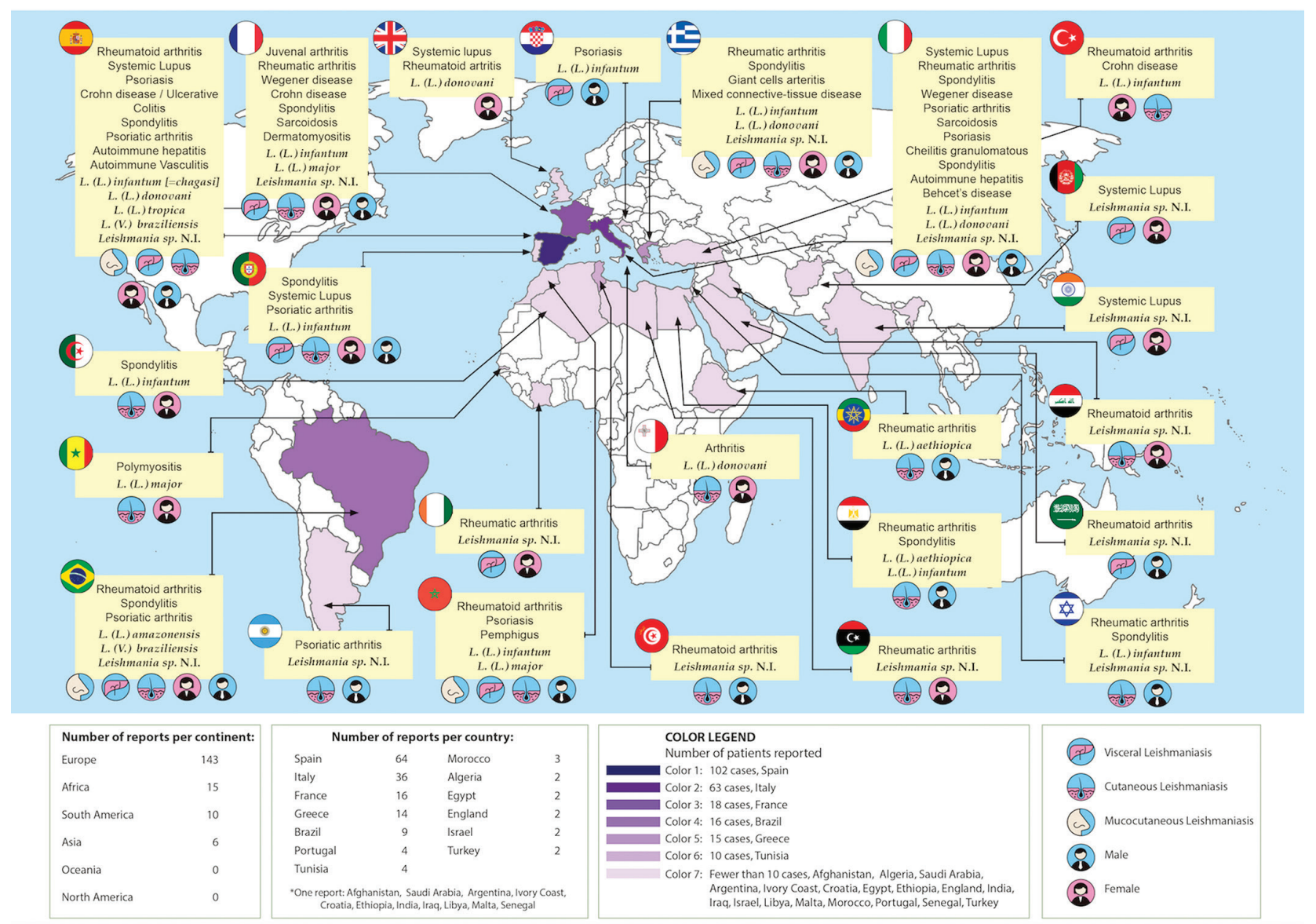

Figure 3 - Geographical distribution of the reported leishmaniasis cases in patients with medication-induced immunosuppression.

Table 2 - The main characteristics of the eight studies that evaluated the prevalence of leishmaniasis in patients treated with immunosuppressive agents for rheumatological, dermatological and gastroenterological diseases.

\begin{tabular}{|c|c|c|c|c|c|}
\hline Authors & Immunosuppressor(S) & Leishmaniasis & Participants & Cases & Prevalence $/ 100,000$ \\
\hline Lequerre et al. ${ }^{15}$ & Anakinra* & Visceral & 35 & 1 & 2,857 \\
\hline Garcia-Vidal et al. ${ }^{13}$ & Infliximab* & Visceral & 94 & 1 & 1,064 \\
\hline Salmon-Ceron et al. ${ }^{18}$ & Anti-TNF* & $\begin{array}{l}\text { Visceral and } \\
\text { cutaneous }\end{array}$ & $57,711 /$ year & 2 & 3 \\
\hline Pérez-Sola et al. ${ }^{14}$ & Anti-TNF & Cutaneous & 6,969 & 2 & 14 \\
\hline Ersozlu Bozkirli et al..$^{12}$ & Anti-TNF & Cutaneous & 142 & 1 & 704 \\
\hline Marie et al. ${ }^{16}$ & $\begin{array}{l}\text { Steroids, methotrexate, } \\
\text { immunobiologicals }\end{array}$ & Cutaneous & 279 & 1 & 358 \\
\hline Cobo-lbáñez et al. ${ }^{11}$ & Anti-TNF & Visceral & 3,166 & 1 & 32 \\
\hline Rubio et al..$^{17}$ & Remsima* & & 78 & 1 & 1,282 \\
\hline Total & & & 68,474 & 10 & 15 \\
\hline
\end{tabular}

${ }^{*}$ Reported co-administration of steroids; TNF = Tumour necrosis factor.

the devastating effects of leishmaniasis in immunosuppressed patients $^{3,20}$. Although immunosuppressive agents provide relevant clinical benefits, they also increase the risk of infection and alter its natural history ${ }^{14,21}$. Interesting data were extracted from the cases of leishmaniasis selected in this study, and a profile of leishmaniasis in patients with medication-induced immunosuppression due to the treatment of rheumatological, dermatological and gastroenterological diseases.

It is also important to elucidate whether there are 


\section{Meta-Analysis Study}

Garcia Vidal et al. ${ }^{13}$

Salmon-Ceron et al. ${ }^{18}$

Pérez-Sola et al. ${ }^{14}$

Ersozlu Bozkirli et al. ${ }^{12}$

Cobo-lbáñez et al. ${ }^{11}$

Rubio et al. ${ }^{17}$

\section{Fixed effect model}

Random effects model

Heterogeneity: $I^{2}=76 \%, \tau^{2}=0.0002$

\section{Events Total}

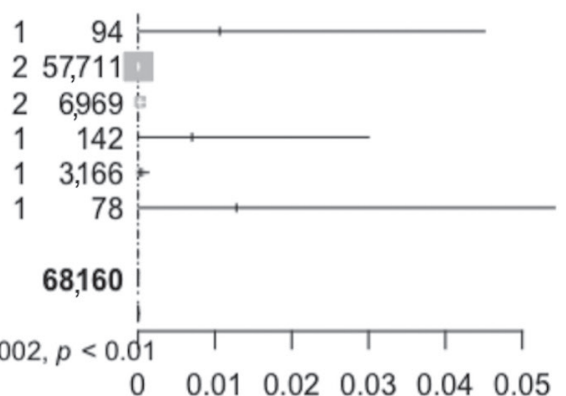

\begin{tabular}{|c|c|c|c|}
\hline oportion & $95 \%-\mathrm{Cl}$ & $\begin{array}{r}\text { Weight } \\
\text { (fixed) }\end{array}$ & $\begin{array}{r}\text { Weight } \\
\text { (random) }\end{array}$ \\
\hline 0.01064 & {$[0 ; 0.04512]$} & 0.1 & 2. \\
\hline 0.00003 & {$[0 ; 0.00010]$} & 84. & $34.8 \%$ \\
\hline 0.00029 & {$[0 ; 0.00086]$} & $10.2 \%$ & $30.4 \%$ \\
\hline 0.00704 & $0 ; 0.03000]$ & 0.2 & $3.9 \%$ \\
\hline 0.00032 & 0 & 4. & $26.0 \%$ \\
\hline 0.01282 & {$[0 ; 0.05423]$} & $0.1 \%$ & $2.3 \%$ \\
\hline & & 100 & \\
\hline .00000 & {$[0 ; 0.00010$} & & 100 \\
\hline
\end{tabular}

Figure 4 - A forest plot showing the effect sizes and confidence intervals of the six methodologically similar studies that assessed the occurrence of leishmaniasis in patients using TNF- $\alpha$ inhibitors. The visual and statistical evaluations showed substantial heterogeneity, making the pooled results unreliable. $\mathrm{Cl}=$ Confidence intervals.

differences in the types and rates of leishmaniasis in patients with different autoimmune conditions. Different underlying pathogenic mechanisms may impair different immune pathways, leading to varying degrees of infection severity ${ }^{11}$. Our results showed that the disease generally developed after $>5$ years of immunosuppression and after 10 years of disease, although earlier development occurred in some cases. The majority of reports involved patients with rheumatological conditions that usually required relatively strong immunosuppression, including the associated use of corticosteroids. It seems clear that chronic and strong perturbations in immune function caused by a combination of immunosuppressors, including lymphocyte and neutrophil dysfunction, defective cellmediated immunity, and decreased immunoglobulin and cytokine production, play important roles in predisposing patients to infections ${ }^{13,18}$.

In contrast to our expectations (Figure 1), most leishmaniasis cases exhibited a typical clinical presentation and responded well to the first course of treatment. This result can be explained by a greater-than-expected frequency of typical cases, as observed in Leishmanial HIV coinfection, but it does not exclude the hypothesis that the number of atypical leishmaniasis cases can be higher in immunosuppressed patients than in the general population. Unfortunately, this cannot be confirmed by the existing literature because no controlled studies were found. Almost all the articles referenced the immediate suspension of immunosuppressive agents after the diagnosis of leishmaniasis, although the reintroduction of immunobiologicals after leishmaniasis was cured was associated with a low frequency of disease reactivation.

The geographical distribution of the included cases also showed unexpected results. The countries that most often reported leishmaniasis in patients with medicationinduced immunosuppression were considerably different from the global picture of this neglected disease in the general population (Figure 3). While leishmaniasis in Europe accounts for only $2 \%$ of all leishmaniasis cases in the world, our article showed that $76.19 \%$ of the cases of leishmaniasis in immunosuppressed individuals were reported in Europe ${ }^{22}$. Chronic rheumatic conditions are more common in developed countries than in developing countries $^{23,24}$, and as a consequence, European countries along the Mediterranean coast have more access to modern immunosuppressive treatments than do countries in South America and the Middle East, in which leishmaniasis is endemic. This result, although worthy of attention, must be interpreted with caution because European countries also have better established monitoring systems, registry systems and publication capacity than South American and African countries, and possible publication bias might exist. Unfortunately, the funnel plot evaluation was limited because of the low number of methodologically similar articles. The absence of a deeper search for grey literature may have contributed to this limitation, although it is probable that no high-quality studies would have been found.

The clinical profile and the geographical distribution of cases, in addition to the fact that reactivation occurred in a minority of cases and that few infected patients were travellers, calls attention to an important epidemiological conclusion. Patients who develop leishmaniasis during medication-induced immunosuppression have usually lived in an endemic region for a long time. Those patients are usually exposed to repeated bites while under immunosuppression, enhancing their chances of developing the disease. This may be reassuring for immunosuppressed patients who seek a better quality of life, as it is likely that sporadic leisure and travel activities in leishmaniasisendemic regions are not associated with a significant risk of leishmaniasis. However, precautionary advice, such as 
avoiding peak vector activity hours and the use of protective clothing, must always be observed.

Only eight articles evaluated the prevalence of leishmaniasis in patients with medication-induced immunosuppression, with a total at-risk population of 68,474. Six studies evaluated patients using anti-TNF immunobiologicals ( $n=68,160$ at risk). Other factors were not evaluated because all the articles reported leishmaniasis as a secondary outcome, meaning that important gaps intended to be filled by this review still exist (Figure 1). This may explain why most articles were scored as "unclear" with regard to the validation methods used, identification of the condition and response rate measurement in the reported quality assessment evaluation. Additionally, we could not evaluate the relative risk of leishmaniasis in patients with medication-induced immunosuppression because no controlled study was included.

In the present study, we conclude that leishmaniasis in patients with medication-induced immunosuppression is a health problem due to the severity of the disease. This condition is reported more frequently in Europe than elsewhere and probably needs specific control measures. Although sporadic activities in endemic regions seem not to be associated with a significantly increased risk of leishmaniasis, patients' education and environmental control are essential prevention measures, which must be reinforced for patients living in endemic areas for leishmaniasis. These measures may enhance the safety of immunosuppression, especially for rheumatological conditions that usually combine anti-TNF therapy with corticosteroids.

We can also conclude that important literature gaps still exist. Studies that specifically seek to analyse leishmaniasis in patients under immunosuppression in large populations $(>1,000)$ must be performed to answer the remaining questions. These questions including the magnitude of risk of developing leishmaniasis in patients under immunosuppression and the measures that immunosuppressed patients should take to avoid leishmaniasis.

\section{AUTHORS' CONTRIBUTIONS}

PSK, FFM, LMHM and CMG conceptualized the study, participated in the investigation, wrote the original draft and participated in the manuscript review and editing. MVC, GD, RNRS, LMTG, CAFS, SSM, and SV participated in the investigation and in manuscript review and editing.

\section{CONFLICT OF INTERESTS}

In the previous 12 months, Dr. Mota served as a consultant for Janssen Biologics, Abbvie, Novartis Corp., Pfizer Inc. and Eli Lilly, received honoraria and received payment for continuing medical education work related to psoriasis that was supported by Eli Lilly, Janssen, Novartis and Abbvie.

\section{FUNDING}

No funding was obtained for this study.

\section{REFERENCES}

1. Rakotonarivo L, Lons-Danic D, Janier M. Plurifocal cutaneous leishmaniasis during treatment with ustekinumab. JAAD Case Rep. 2018;4:298-300.

2. Name RQ, Borges KT, Nogueira LS, Sampaio JH, Tauil PL, Sampaio RN. Estudo clínico, epidemiológico e terapêutico de 402 pacientes com leishmaniose tegumentar americana atendidos no Hospital Universitário de Brasília, DF, Brasil. An Bras Dermatol. 2005;80:249-54.

3. Carranza-Tamayo CO, Assis TS, Neri AT, Cupolillo E, Rabello A, Romero GA. Prevalence of Leishmania infection in adult HIV/AIDS patients treated in a tertiary-level care center in Brasilia, Federal District, Brazil. Trans R Soc Trop Med Hyg. 2009;103: 743-8.

4. van Griensven J, Carrillo E, López-Vélez R, Lynen L, Moreno J. Leishmaniasis in immunosupressed individuals. Clin Microbiol Infect. 2014;20:286-99.

5. Arens K, Filippis C, Kleinfelder H, Goetzee A, Reichmann G, Crauwels P, et al. Anti-tumor necrosis factor $\alpha$ therapeutics differentially affect Leishmania infection of human macrophages. Front Immunol. 2018;9:1772.

6. Joanna Briggs Institute. The Joanna Briggs Institute reviewers' manual, 2014. Adelaide: Joanna Briggs Institute; 2014.

7. Schwarzer G. Meta: an R package for meta-analysis. R News. 2007;7:40-5.

8. Viechtbauer W. Conducting meta-analyses in $\mathrm{R}$ with the metafor package. J Stat Softw. 2010;36:1-48.

9. Weightr: estimating weight-function models for publication bias. [cited 2020 April 17]. Available from: https://CRAN.R-project. org/package=weightr

10. Darcis G, Van der Auwera G, Giot JB, Hayette MP, Tassin F, Arrese Estrada J, et al. Recurrence of visceral and mucocutaneous leishmaniasis in a patient under immunosuppressive therapy. BMC Infect Dis. 2017;17:478.

11. Cobo-Ibáñez T, Descalzo MÁ, Loza-Santamaría E, Carmona L, Muñoz-Fernández S. Serious infections in patients with rheumatoid arthritis and other immune-mediated connective tissue diseases exposed to anti-TNF or rituximab: data from the Spanish registry BIOBADASER 2.0. Rheumatol Int. 2014;34:953-61. 
12. Ersozlu Bozkirli D, Turunc T, Gorek Dilektasli A, Yucel E. Granulomatous diseases following treatment with anti-TNF therapies. Clin Microbiol Infect. 2011;17 Suppl:S411-2.

13. Garcia-Vidal C, Rodríguez-Fernández S, Teijón S, Esteve M, Rodríguez-Carballeira M, Lacasa JM, et al. Risk factors for opportunistic infections in infliximab-treated patients: the importance of screening in prevention. Eur J Clin Microbiol Infect Dis. 2009;28:331-7.

14. Pérez-Sola MJ, Torre-Cisneros J, Pérez-Zafrilla B, Carmona L, Descalzo MA, Gómez-Reino JJ, et al. Infections in patients treated with tumor necrosis factor antagonists: incidence, etiology and mortality in the BIOBADASER registry. Med Clin (Barc). 2011;137:533-40.

15. Lequerré T, Quartier P, Rosellini D, Alaoui F, De Bandt M, Mejjad $\mathrm{O}$, et al. Interleukin-1 receptor antagonist (anakinra) treatment in patients with systemic-onset juvenile idiopathic arthritis or adult onset Still disease: preliminary experience in France. Ann Rheum Dis. 2008;67:302-8.

16. Marie I, Ménard JF, Hachulla E, Chérin P, Benveniste O, Tiev K, et al. Infectious complications in polymyositis and dermatomyositis: a series of 279 patients. Semin Arthritis Rheum. 2011;41:48-60.

17. Rubio E, Ruiz A, López J, López-Chozas JM, Bermudez L, Aguilera C, et al. Prospective study of 78 patients treated with infliximab biosimilar Remsima ${ }^{\circledR}$. Ann Rheum Dis. 2016;75 Suppl 2:1006.
18. Salmon-Ceron D, Tubach F, Lortholary O, Chosidow O, Bretagne $\mathrm{S}$, Nicolas N, et al. Drug-specific risk of non-tuberculosis opportunistic infections in patients receiving anti-TNF therapy reported to the 3-year prospective French RATIO registry. Ann Rheum Dis. 2011;70:616-23.

19. Sedgwick P, Marston L. How to read a funnel plot in a metaanalysis. BMJ. 2015; 351:h4718.

20. Ramos JM, León R, Merino E, Montero M, Aljibe A, Blanes M, et al. Is Visceral leishmaniasis different in immunocompromised patients without human immunodeficiency virus? A comparative, multicenter retrospective cohort analysis. Am J Trop Med Hyg. 2017;97:1127-33.

21. De Leonardis F, Govoni M, Lo Monaco A, Trotta F. Visceral leishmaniasis and anti-TNF-alpha therapy: case report and review of the literature. Clin Exp Rheumatol. 2009;27:503-6.

22. World Health Organization. Leishmaniasis in the WHO European region. [cited 2020 April 18]. Available from: http://www. euro.who.int/_data/assets/pdf_file/0007/246166/Fact-sheetLeishmaniasis-Eng.pdf?ua=1

23. Rudan I, Sidhu S, Papana A, Meng S, Xin-Wei Y, Wang W, et al. Prevalence of rheumatoid arthritis in low- and middle-income countries: a systematic review and analysis. J Glob Health. 2015;5:010409.

24. Mariller MM, Santos-Eggimann B. The prevalence of rheumatic diseases in the elderly in developed countries and its evolution over time. Soz Praventivmed. 2005;50:45-51. 